\title{
Enhancement of UnderwaterImages using Color Balancing and Multi-Scale Fusion Technique
}

\author{
B Raja Sekhar, M Shiva Kumar
}

\begin{abstract}
The method proposed in this paper is efficient and does not need any external hardware nor does it need information regarding the conditions and structure of the scene which is to be enhanced after being captured in underwater as it is a degraded image due to presence of scattering and absorption intensity due to underwater particles and light. Two images, which are obtained after white balancing and color compensation of the degraded input image, are combined and the weight maps of the two images help in enhancement of edges and color contrast of the final output image. The creation of weight maps lead to the low frequency component of the recombined image having artifacts in it. Multiscale fusion technique has also been adopted in this paper. On making a comparative analysis, it was observed that the proposedmethod leads to enhancement of underwater images and videos in such a way that the dark regions exposition is improved, global contrast is better and edges are sharper.
\end{abstract}

Index Terms - white balancing, Multiscale fusion, underwater images.

\section{INTRODUCTION}

The underwater environment of many rare attractions like marine animals and fishes, amazing landscape, and mysterious shipwrecks. An important source of underwater photography and underwater imaging [1] etc. are interest in different branches of technology, for example inspection of underwater infrastructures and cables [3], control of underwater

Vehicles [2], detection of man-made objects, marine biology research [6] and archeology.

For the image enhancement method [8] are three different types. Such as point processing, spatial filtering and image coloring. Undertaking of point processing are three different technique like contrast enhancement, histogram modeling, image averaging. The spatial filtering is four different techniques such as linear filters, non linear filters, edge detection, and zooming. And the image coloring there are two different techniques like pseudo coloring, false coloring.

The term in radiology of contrast enhancement is a ubiquitous as well as can be used in three different types. (1) It may refer two methods of administering contrast agent on a scan by exaggerating the visible difference between adjacent structures. The histograms of image represent the relative frequency occurrence of grey levels in image. The modify an image globally as well as histogram has a desired shape because are used in histogram modeling techniques The techniques are digital image processing is an image averaging of an often employed to enhance video images have been corrupted by random noise.

Linear filters process time varying input signals to produces output signals and used to the subject constraint of linearity. In this result shown from system composed digital algorithm classified having a linear response. The output is not a linear function it's an input because of this non linear filter. The filters of output signals $\mathrm{R}$ as well as $\mathrm{S}$ for the input signals $\mathrm{r}$ as well as $\mathrm{s}$ are separately. The input are a linear combination $\alpha r+\beta s$ but does not output always $\alpha R+$ $\beta S$. Image processing technique is edge detection if finding the boundaries of an object with an image. In edge detection is used for image segmentation data extraction like a computer vision, machine vision and image Processing etc.

To the gray values based on a specific criteria used of an assigning different colors this colors are called pseudo coloring. The monochrome images from process associated with true color image because is used to differentiate of assigning colors. In refers to methods of a group color rendering are used to display image in color is known as false color or pseudo color. There are two types of electromagnetic spectrum i.e. visible part or non visible part.

\section{LITERATURE SURVEY}

M. D. Kocaket.al, proposed in their paper a 3D reconstruction method in underwater environments are presented along with a review of optical sensors. The different sensors hardware that makes them possible and this technique are used to obtain range data have been listed as well as explained. A classification has been proposed the development of existing solution is reviewed in literature. In this topic has been gathered as well as considered new developments are commercial solutions as well as previous reviews. The underwater applications is non-trivial are used to be 3D sensing system. The basics feature considered are: (1) the payload volume, in case the system is an on board platform, weight and power available. (2) the budget (3) the measurement time and (4) the expected quality of the data gathered. Optical sensors, are very sensitive to water turbidity and surface texture. 


\section{ENHANCEMENT OF UNDERWATERIMAGES USING COLOR BALANCING AND MULTI-SCALE FUSION TECHNIQUE}

G. L. Foresti in this paper described a vision based system for the inspections of underwater structures, for e.g. pipelines, cables, etc. by used an autonomous underwater vehicle (AUV). Generally, underwater overview is execute by remote operated vehicles (ROVs) a support vessels by a human operators. The proposed system allowed the autonomous underwater vehicle to the accomplish in autonomy. However, the extended kalam filter, use of the three-dimensional model of environment, for the control of the vehicle in real time. The proposed method its efficiency (I) case of critical as well as complex situations because on real underwater picture has a demonstrated the validity.

A. Ortizet.al, proposed in their paper the surveillance as well as inspection of underwater installations, likes a power or telecommunication cables as well as pipelines, its carried out by the, living on the surface, drive a remotely operated vehicl as cameras mounted it. The easily prone errors because the loss of attention or fatigue of human operator, as well as this condition are tedious or high time-consuming task. Too, Increased complexity the lack of quality and typical seabed images, it is characterized by blurring, nonuniform illumination, instability in the vehicle motion as well as lack of contrast. The proposed method tracks the average success rate above $90 \%$ because its vision system.

A. Olmos and E. Trucco, proposed a system for detecting the presence of unconstrained man-made objects in unconstrained subsea videos. Which are reasonably stable features in underwater imaginary because the classification is based on contours. There are two types of contours. (1) Whether the image contains man-made objects because a two-features Bayesian classifier. (2) the system determines automatically an optimal scale for contour extraction by optimizing a quality metric. This features used to capture general properties of man-made structures used to measures inspired by perceptual organization. The system classified approximately 1390 test images from the types of five different underwater videos, poor quality as well as generality of the classification task 1 , in spite of the varying images contents.

Y. Kahanov and J. G. Royal, stated that the significant element of revealed in $7^{\text {th }}$-century AD shipwreck, Dor D because closely spaced set of unpegged mortise as well as tenon joints. As well as the additional wrecks because it establishes a better database for ship construction in $4^{\text {th }}$ centuries $\mathrm{AD}$ because its provide an additional information for transitional period of ship building in Mediterranean. The more complicated images of general evolutionary trend, have been considered as a dis-appearing technique because they present some character that have traditionally.

\section{PROPOSED METHOD}

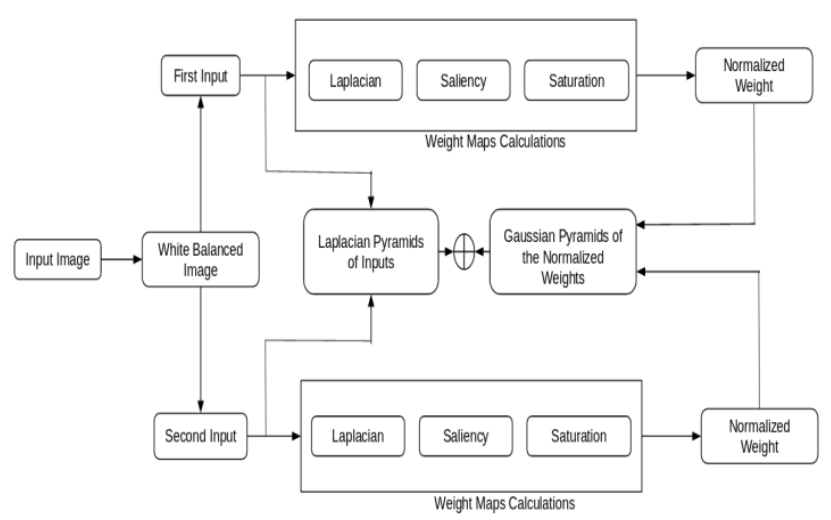

Fig. Block Diagram of the Proposed Method

\section{A. Pre-Processing Of Input Images}

(1) In underwater images, there is a problem of color casts because of the different illuminations. Hence, to remove this effect in images, color correction is required and it is done by white-balancing the original input image.

(2) The first input is obtained by using gamma correction technique on the white balanced image. This is done in order to adjust the global contrast of the image because the whitebalanced image looks too bright. Moreover, when the image is taken underwater at depths of more than $30 \mathrm{ft}$, the colors seen in the white-balanced image are not perfect because some absorbed colors cannot be properly recovered.

(3) Gamma correction improves contrast between light and dark regions of images, but it also leads to some details in the over or under exposed regions being lost. In order to correct this, a second input is taken by sharpening the white balanced image. The sharpening method used is a normalized unsharp masking technique. This method does not require tuning of any parameters and is seen to be an effective sharpening technique. It helps to reduce degradation of the image due to scattering effects. But there can still be some artifacts present in this image due to highfrequency noise present in it. Therefore, the next step is to apply multi-scale fusion of the images to remove these artifacts.

\section{B Multi-Scale Fusion Process}

(1) For the multi-scale fusion process, the weight maps of images are used. In order to make weight maps, pixels with high weight values are used. The weight maps are made using three metrics or processes, namely, Laplacian contrast weight, Saliency weight and Saturation weight.

(2) Laplacian contrast weight: In this weight map absolute value of Laplacian filter is applied on each of the luminance channel so as to estimate global contrast of the image. However, this weight map is not enough for dehazing underwater images because it does not give difference between flat and ramp regions in images. Therefore, other metrics are also used. 
(3) Saliency weight: Some salient objects present in the underwater images might lose their intensity levels, hence to emphasize them, saliency weight map is used. This saliency map has a tendency to highlight areas with high luminance values, and hence in order to overcome this another weight map is also used in combination with this one.

(4) Saturation Weight: In this weight map, highly saturated regions are advanced which allows the fusion algorithm to get adapted to chromatic information. This weight map is computed by taking the deviation of every pixel location between the red, green and blue color channels and also the luminance value of the input.

(5) Normalized Weights:After the individual weight maps are computed, they are merged into a single weight map by summing the three maps. The normalization process of the aggregated weight map includes pixel-per-pixel normalization and then each pixel's weight in each map is divided by he sum of weights of same pixel over all maps.

(6) Multi-scale Fusion Process:Laplacian and Gaussian pyramid methods are used for multiscale decomposition of the images. In Laplacian pyramid decomposition of each of the source input, each image is decomposed into sum of bandpass images. Gaussian pyramid is used to decompose the normalized weight map images. The number of levels of decomposition in both the pyramids is same. At each level, the laplacian inputs and Gaussian normalized weights are mixed independently. The number of levels selected depends on the size of the image and this choice impacts the visual quality of the combined image. The fused images at all levels are then summed to get the dehazed output after up sampling is done appropriately.

\section{RESULT ANALYSIS}

In this work we gone through process of single image dehazing which leads for image enhancement. To improve the quality of underground image there is need of some important steps to be followed.The result analysis is done using MATLAB 2016a software.Analysis is done by scripting the code in matlab and results proves that our method is better for different conditions of haze and underwater problems.

For the input image white balancing is applied at the first step, after that the output image is passed through gamma correction and edge sharpening. The output of gamma correction and edge sharpening are fused by multiscale fusion to get final enhanced image.

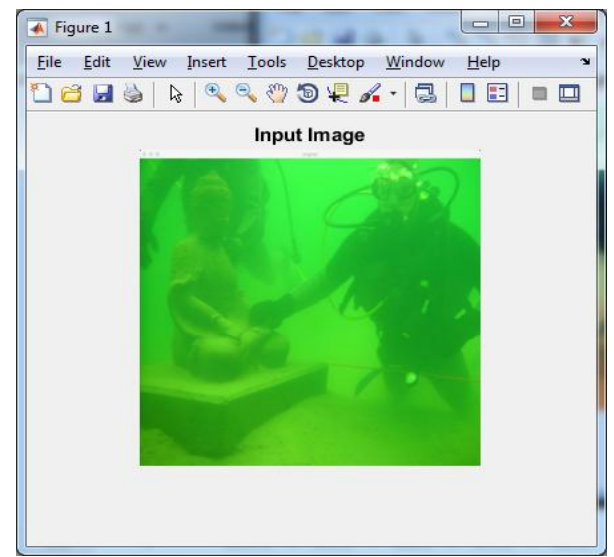

Fig.1 Input Image for Enhancement
Here input image is selected which is underwater image and present haze in it. To remove the haze and make it more enhanced image we will apply white balancing. Input image is took from underwater and degraded because of absorption as well as scattering problems.

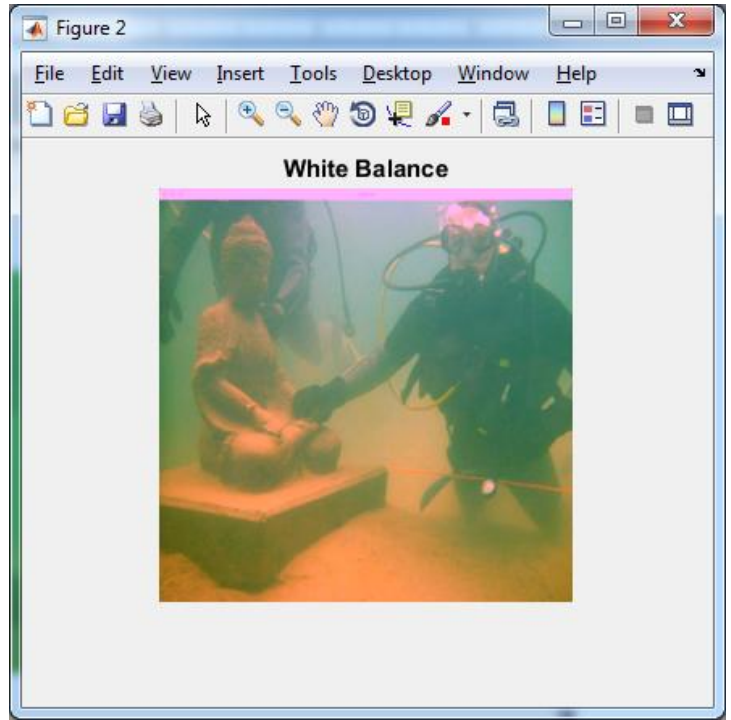

Fig.2 White balance Image

White balancing is used to remove the particular color caste induced because of scattering effects. White balance image mostly enhance the edges in image and improve the details present in the scene with removing back scattering which cause the loss of contrast.

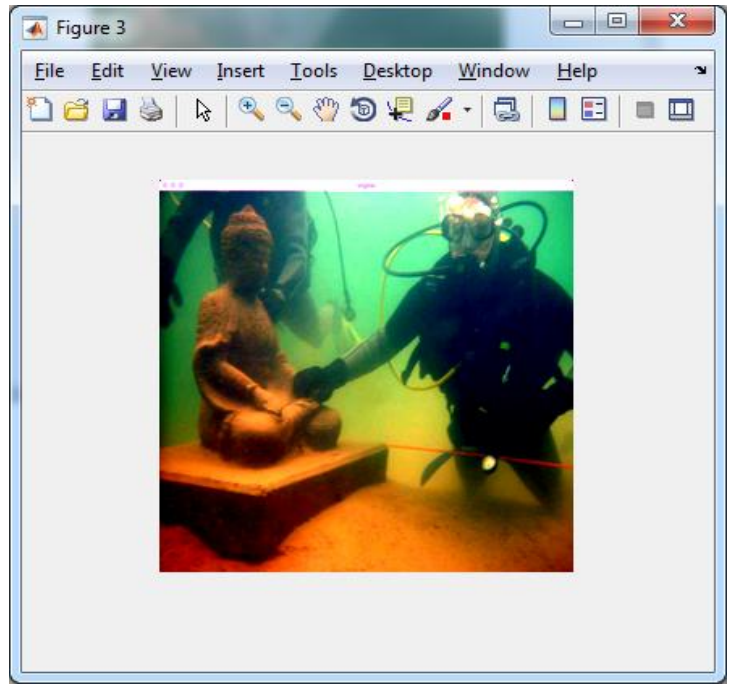

Fig.3 Edge Sharpening applied to an Image

To overcome loss problems we applied edge sharpening and it preserves the quality of an input image. 


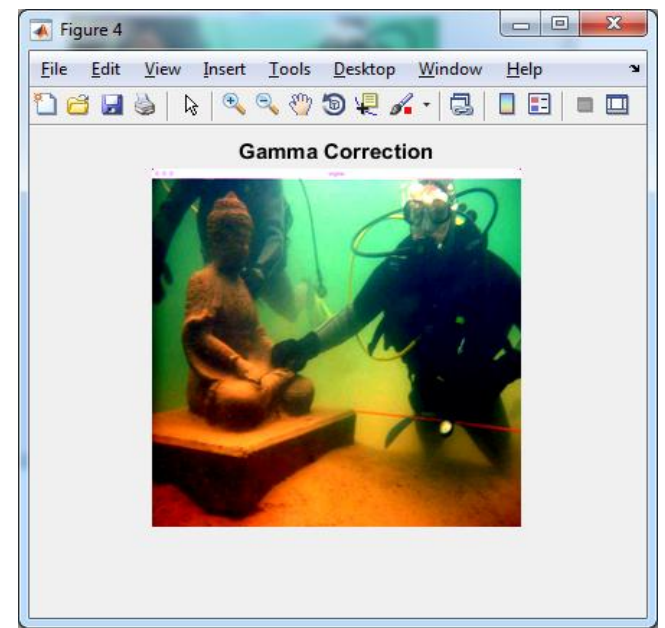

Fig.4 Gamma Correction Applied For Image

Gamma corrections are mostly used for improving the global contrast because underwater images are too much bright.

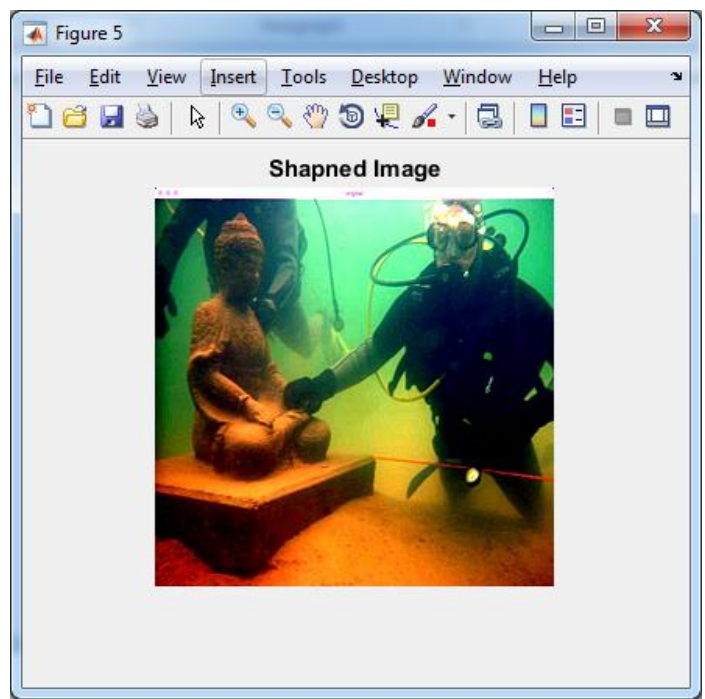

Fig.5 Sharpened Image

Compensation with loss is done by using sharpening of the white balanced image.

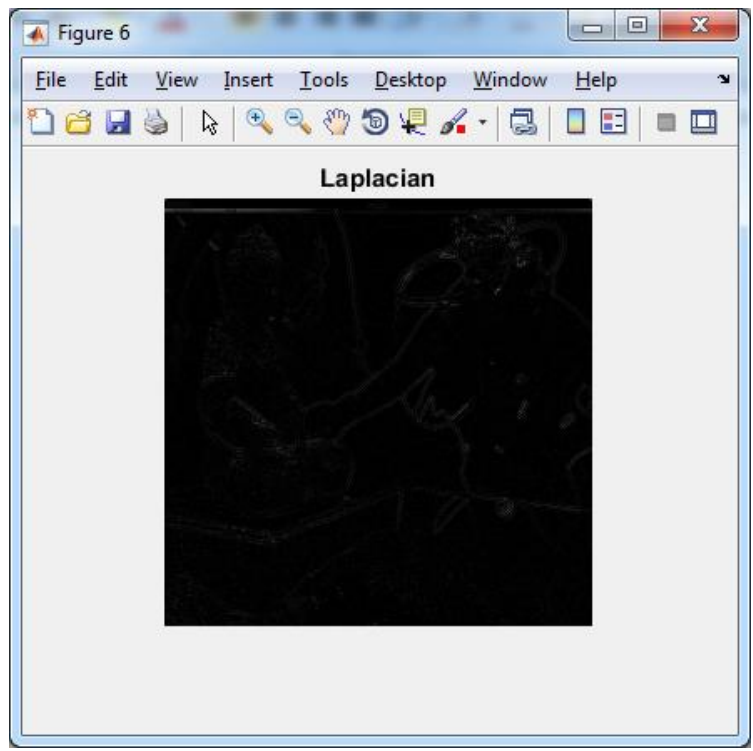

Fig.6 Laplacian Image

There is application of laplacian filter on input luminance channel to get the global contrast of input image.

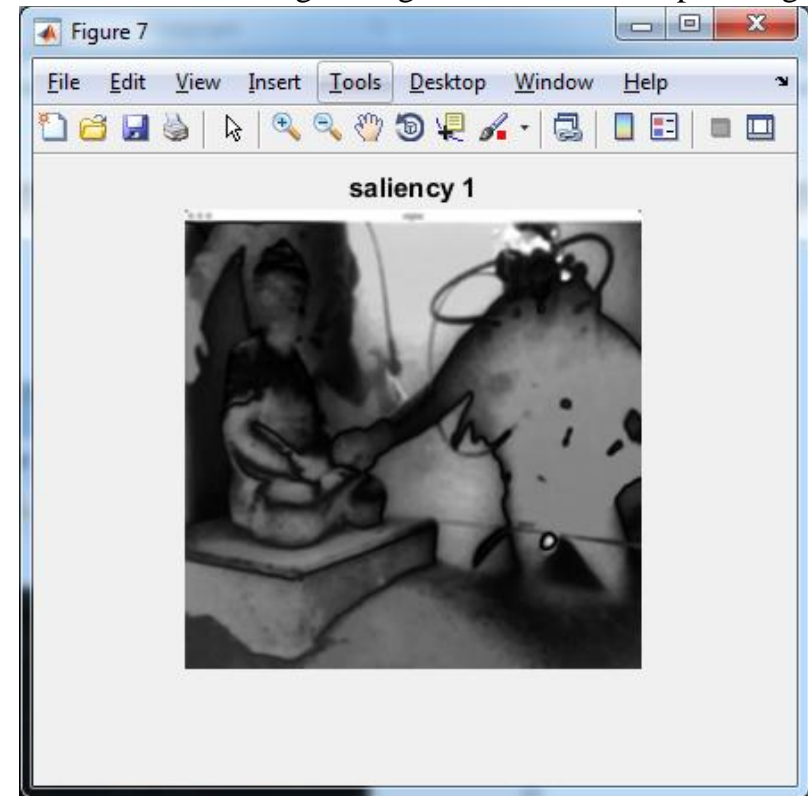

Fig.7 Saliency Detected Image for Saliency 1

Saliency region is nothing but important region in an image. This technique is applied for gamma corrected image.

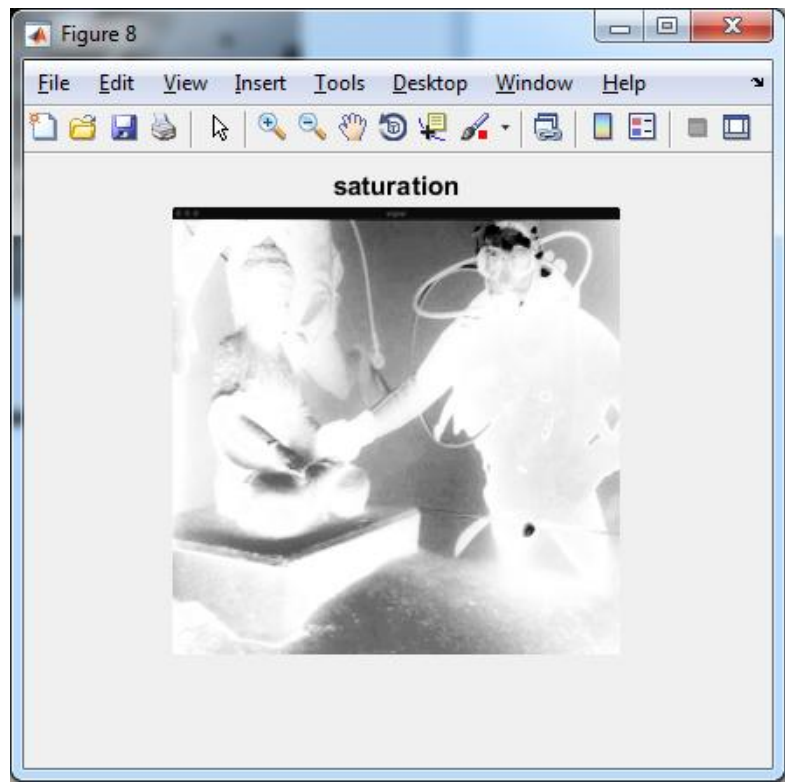

Fig.8 Saturated Image (1st Image)

The information from green channel should not mix with the information of the red channel which means that there should not be red loss compensation. This technique is applied for gamma corrected image.

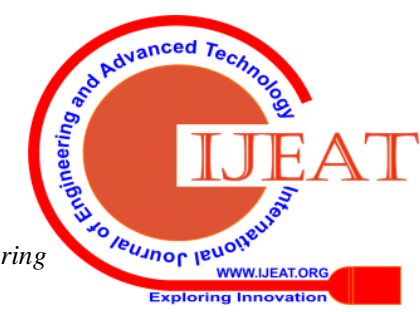




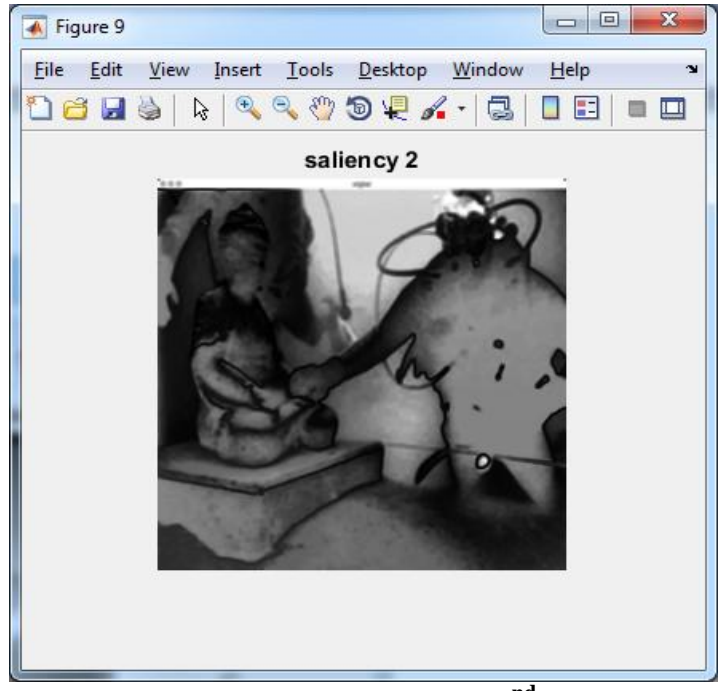

Fig.9 Saliency Detection in $2^{\text {nd }}$ image

Saliency region is nothing but important region in an image. This technique is applied for edge sharpened image.

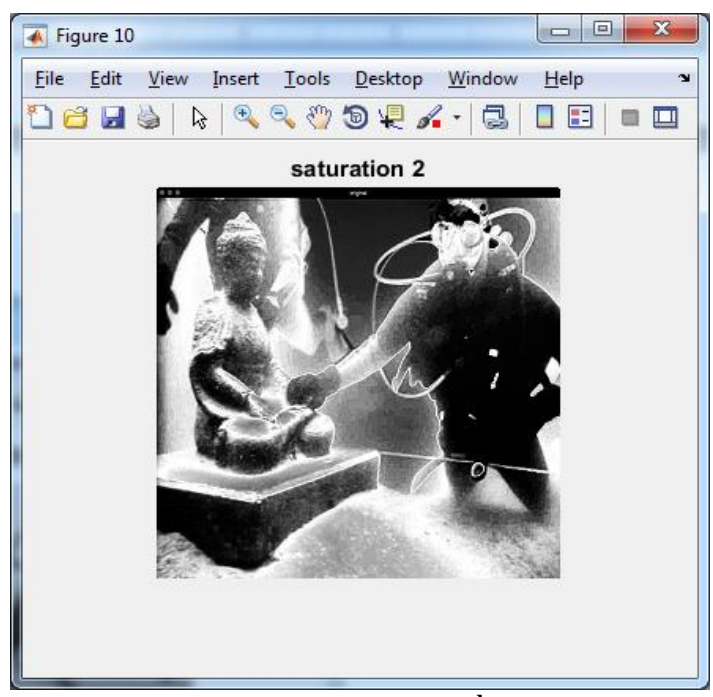

Fig.10 Saturation in $2^{\text {nd }}$ Image

This technique is applied for the edge sharpened image. By highly saturated region there is advantage of getting highly chromatic information.

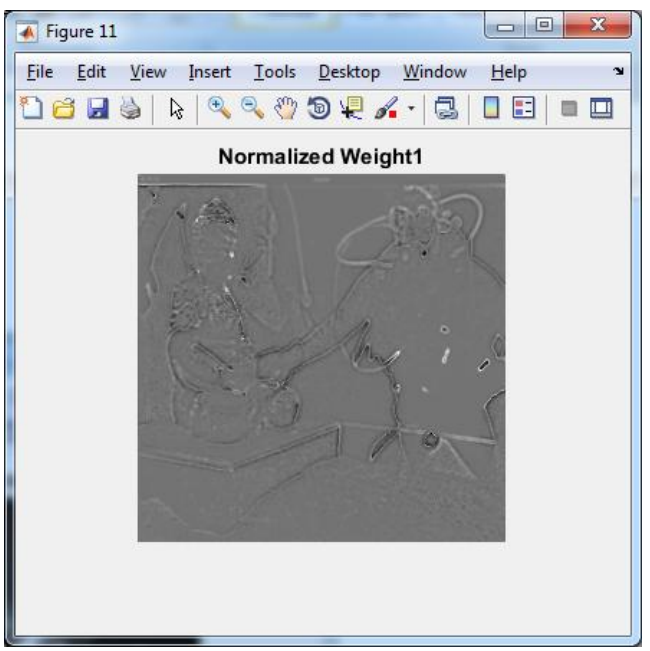

Fig.11 Normalized Weight applied for $1^{\text {st }}$ image
Normalized weight technique is applied for the steps applied at gamma corrected image.

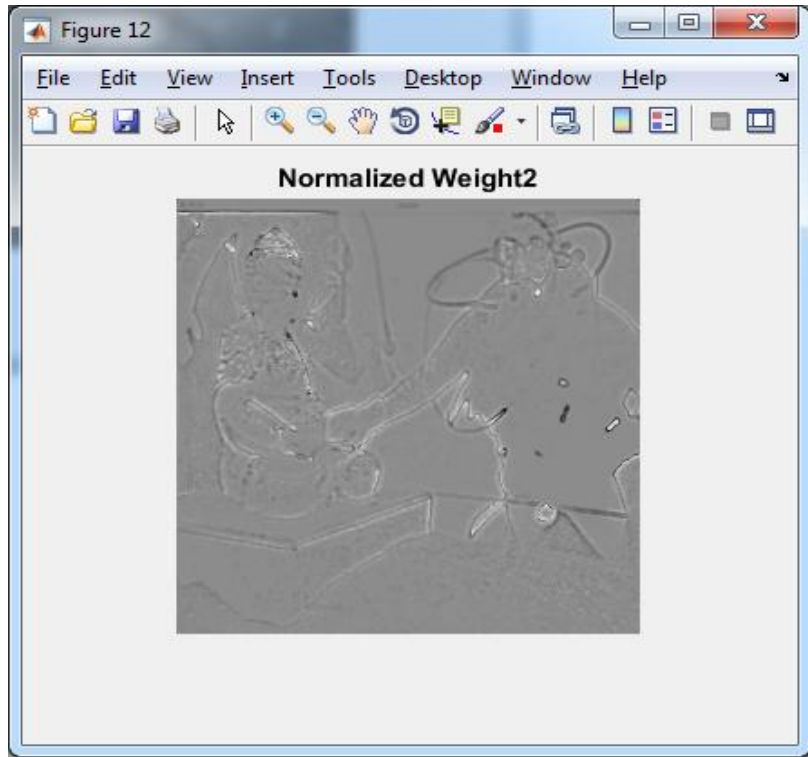

Fig. 12 Normalized Weight applied for $2^{\text {nd }}$ image

Normalized weight technique is applied for the steps applied at edge sharpened image. By applying weights to particular pixels the normalized weight map is created to reconstruct the original image.

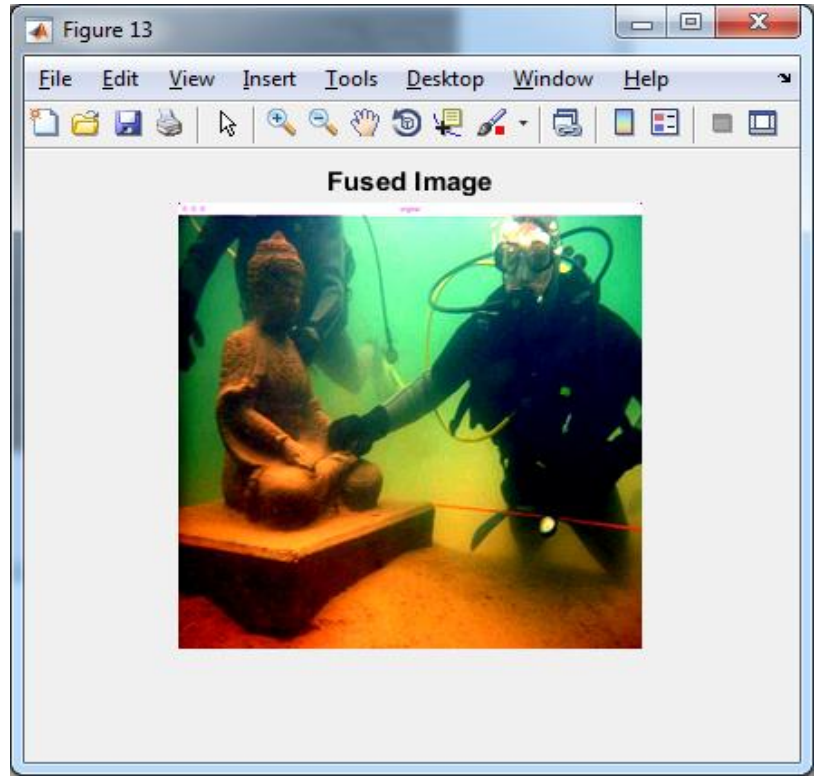

Fig.13 Final Fused Image (Enhanced Image)

Final fused image is obtained by following steps of proposed work. Final fused image is free from haze and removed scattering effects from underwater image.

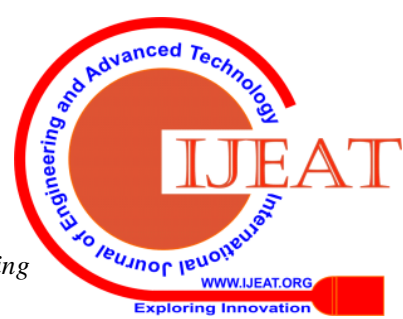




\section{CONCLUSION}

A novel method for enhancement of videos and images [8], taken underwater, was introduced in this paper. The technique requires use of fusion method and the original image and no other information is necessary. Through quantitative analysis, it has been shown that the method introduced can enhance various ranges in which the images have been taken underwater with better accuracy. The method has also proven its ability in recovering edges and features that are faded. We proved that our proposed work will provide better solution for image captured in underwater and which is hazed and present with scattering effects.

\section{REFERENCES}

1. M. D. Kocak, F. R. Dalgleish, M. F. Caimi, and Y. Y. Schechner,"A focus on recent developments and trends in underwater imaging,"Marine Technol. Soc. J., vol. 42, no. 1, pp. 52-67, 2008.

2. G. L. Foresti, "Visual inspection of sea bottom structures by anautonomous underwater vehicle," IEEE Trans. Syst., Man, Cybern. B,Cybern., vol. 31, no. 5, pp. 691-705, Oct. 2001.

3. A. Ortiz, M. Simó, and G. Oliver, "A vision system for an underwater cable tracker," Mach. Vis. Appl., vol. 13, pp. 129 140, Jul. 2002.

4. A. Olmos and E. Trucco, "Detecting man-made objects in unconstrained subsea videos," in Proc. BMVC, Sep. 2002, pp. $1-10$.

5. B. A. Levedahl and L. Silverberg, "Control of underwater vehicles infull unsteady flow," IEEE J. Ocean. Eng., vol. 34, no. 4, pp. 656-668,Oct. 2009.

6. C. H. Mazel, "In situ measurement of reflectance and fluorescencespectra to support hyperspectral remote sensing and marine biologyresearch," in Proc. IEEE OCEANS, Sep. 2006, pp. 1-4.

7. Y. Kahanov and J. G. Royal, "Analysis of hull remains of the DorD Vessel, Tantura Lagoon, Israel," Int. J. Nautical Archeol., vol. 30,pp. 257-265, Oct. 2001.

8. R. Schettini and S. Corchs, "Underwater image processing: state of theart of restoration and image enhancement methods," EURASIP J. Adv.Signal Process., vol. 2010, Dec. 2010, Art. no. 746052.

9. S. G. Narasimhan and S. K. Nayar, "Contrast restoration of weather degraded images," IEEE Trans. Pattern Anal. Mach. Learn., vol. 25,no. 6, pp. 713-724, Jun. 2003.

10. D.-M. He and G. G. L. Seet, "Divergent-beam LiDAR imaging in turbid water," Opt. Lasers Eng., vol. 41, pp. 217 231, Jan. 2004.

11. Y. Y. Schechner and Y. Averbuch, "Regularized image recovery inscattering media," IEEE Trans. Pattern Anal. Mach. Intell., vol. 29, no. 9,pp. 1655-1660, Sep. 2007.

12. B. L. McGlamery, "A computer model for underwater camera systems,”Proc. SPIE, vol. 208, pp. 221-231, Oct. 1979.

13. J. S. Jaffe, "Computer modeling and the design of optimal underwaterimaging systems," IEEE J. Ocean. Eng., vol. 15, no. 2, pp. 101-111,Apr. 1990.

14. H. Koschmieder, "Theorie der horizontalensichtweite," BeitragePhys.Freien Atmos., vol. 12, pp. 171-181, 1924.

15. M. Levoy, B. Chen, V. Vaish, M. Horowitz, I. McDowall, and M. Bolas, "Synthetic aperture confocal imaging," in Proc. ACM SIGGRAPH,Aug. 2004, pp. 825-834. 